\title{
Attitudes and beliefs of emergency physicians on lab tests utilization in clinical practice. A multicenter survey
}

\author{
Carlotta Saccenti, ${ }^{1}$ Marco Lunian, ${ }^{1}$ Martina Montagnana, ${ }^{2}$ Andrea Balloni, ${ }^{3}$ Giulia Maria Azin, ${ }^{4}$ \\ Roberto Lerza, ${ }^{5}$ Michele Mitaritonno, ${ }^{6}$ Matteo Pistorello, ${ }^{7}$ Michele Santoro, ${ }^{8}$ Roberto Fiorini, ${ }^{9}$ \\ Laura Bonfanti ${ }^{1}$
}

${ }^{1}$ Emergency Department, University Hospital of Parma, Parma; ${ }^{2}$ Section of Clinical Chemistry, University of Verona, Verona; ${ }^{3}$ Emergency Department, ASUR Marche Hospital of Fermo, Fermo; ${ }^{4}$ Emergency Department, Niguarda Hospital, Milan; ${ }^{5}$ Emergency Department, S. Paolo Hospital, Savona; ${ }^{6}$ Emergency Department, Hospital of Cosenza, Cosenza; ${ }^{7}$ Emergency Department, Hospital of Treviso, Treviso; ${ }^{8}$ Emergency Department, “Cardinal Giovanni Panico” Hospital, Tricase, Lecce; ${ }^{9}$ Emergency Department, Hospital of Vaio-Fidenza, Fidenza, Italy

\begin{abstract}
It is commonly perceived that the main obstacle for an effective partnership between Emergency Medicine (EM) and Laboratory Medicine (LM) is the lack of reciprocal knowledge and understanding. A further aspect is a partial comprehension of clinical significance and implications of some tests. The real scenario of attitudes and beliefs of Emergency Physicians (EPs) on lab tests utilization in clinical practice remains largely unclear. Therefore, this original study was designed to explore this field of medical knowledge, by using a survey on attitudes and beliefs in laboratory diagnostics which was disseminated among many Italian EPs. A questionnaire containing 20 different items/statements was designed and then disseminated either directly, or in a digital format. Overall, 183 questionnaires were compiled and collected. The results of this nationwide survey seem to confirm the existence of a suboptimal knowledge of both clinical and economic impact of urgent laboratory tests by Italian EPs. An unsatisfactory relation-
\end{abstract}

Correspondence: Laura Bonfanti, Emergency Department, University Hospital of Parma, Via Gramsci 14, 43126 Parma, Italy.

E-mail: labonfanti@ao.pr.it

Key words: Emergency medicine; Laboratory tests; Clinical biochemistry; Diagnostic process; Diagnostic strategies; Emergency Department.

Conflict of interest: the authors declare no potential conflict of interest.

Funding: none.

Received for publication: 16 April 2019.

Revision received: 24 July 2019.

Accepted for publication: 8 August 2019.

This work is licensed under a Creative Commons Attribution 4.0 License (by-nc 4.0).

(Copyright: the Author(s), 2019

Licensee PAGEPress, Italy

Emergency Care Journal 2019; 15:8237

doi:10.4081/ecj.2019.8237 ship between EPs and laboratory professionals seems also to emerge. Several EPs attribute this to Shortage of time to think (18.6\%), followed by Fair of medical-legal litigation (13.6\%). On the other hand, interestingly, it also emerged that some Italian hospitals still include obsolete o redundant tests in panels of urgent laboratory analyses, potentially misleading for the diagnostic reasoning in the Emergency Department (ED). In conclusion, the virtuous circle between EM and LM should be further reinforced for optimizing clinical pathways in Italian EDs, improving clinical outcomes and reducing unnecessary expenditures. A major agreement between laboratory and emergency professionals for harmonization of urgent test panels seems advisable.

\section{Introduction}

According to some published data, laboratory testing participates to the vast majority of clinical decisions. ${ }^{1,2}$ Since rapid and accurate diagnoses followed by appropriate treatments are the mainstays in the field of Emergency Medicine (EM), the Laboratory Medicine (LM) shall modulate its actual organization to fulfill these tasks and hence provide rapid and accurate tests that may allow Emergency Physicians (EPs) to make early rule out or rule in of most urgent and acute disorders. ${ }^{3}$

Two Italian scientific societies of EM (Academy of Emergency Medicine and Care, AcEMC) and LM (Italian Society of Clinical Biochemistry and Clinical Molecular Biology, SIBioC) have been precursor for establishing a fruitful collaboration, with publication of many consensus documents on important topics such as panels of urgent diagnostic tests, ${ }^{4,5}$ diagnosing acute coronary syndrome, ${ }^{6}$ sepsis, ${ }^{7}$ and venous thromboembolism. ${ }^{8}$

It is commonly perceived that a main obstacle for an effective partnership between EM and LM is the lack of sufficient reciprocal knowledge and understanding. A further aspect contributing to disrupt the liaison between EM and LM is an occasionally partial comprehension of clinical significance and implications of some tests. Notably, misconceptions and false myths are still exceptionally common in clinics, as comprehensively discussed elsewhere. ${ }^{9,10}$

In particular, appropriateness of test ordering remains a matter of major concern, whilst reliable evidence suggests that the panels of urgent tests are quite variegated, so highlighting a substantially unstandardized scenario..$^{9,11,12}$ A recent multicenter Spanish study, which has involved 36 hospitals, showed that the rate of stat tests 
requests was comprised between 44-412 per 1000 ED patient admissions. More importantly, the authors of this article also concluded that Spanish hospitals would have saved $>1$ million unnecessary tests by simply harmonizing the diagnostic approaches and establishing a favorable interdepartmental cooperation between EPs and laboratory professionals. ${ }^{13}$

Despite some reliable guidance on appropriateness of laboratory test requests in the $\mathrm{ED}$ has been published by $\mathrm{SIBioC},{ }^{14}$ then mutually agreed by both SIBioC and AcEMC, ${ }^{4}$ the real scenario of attitudes and beliefs of EPs on lab tests utilization in clinical practice remains largely unclear. Therefore, this original study was designed to explore this field of medical knowledge, by using a survey on attitudes and beliefs in laboratory diagnostics which was disseminated among many Italian EPs.

\section{Materials and Methods}

\section{Design}

A questionnaire containing 20 different items/statements (shown in the Appendix) was designed by a group of members of both national societies (AcEMC and SIBioC), which was then disseminated either directly (by a chief or senior physician), or in a digital format available on the AcEMC website to a large number of Italians EPs. The survey contained questions on inclination in requesting and managing laboratory medicine testing, specific knowledge on clinical meaning of some laboratory tests and/or profiles, and some economic and organizational implications of in vitro diagnostic testing. Some questions allowed a single response (e.g., yes or no), whilst others allowed multiple responses. All participants were informed that the questionnaire was anonymous, was only aimed for scientific purposes, and that participation was not mandatory.

\section{Data collection}

All responses were collected anonymously. In each of the 15 centers involved, a physician was responsible for distribution and collection of questionnaires, as well as for returning the surveys to the AcEMC administrative assistant. All the questionnaires compiled trough the digital platform were collected by one of the Authors (LB). The available period for compiling the questionnaires was comprised between February 1st and March 15th, 2019. After collection was concluded, both manual and digital questionnaires were pooled and analyzed altogether. The study was performed in agreement with the ethical standards established by the Helsinki Declaration of 1975. Due to the type of study, approval by ethic committee was not necessary (participation was open and free).

\section{Results}

Overall, 183 questionnaires were compiled and collected. Since not all the responders answered to all the 20 items, the sum of responses was $<183$ in some cases.

To the first statement Generally, when you order lab tests, 97 (53\%) responders chose I only select the test I consider useful in that clinical presentation, whereas $86(47 \%)$ chose I use standard panels. The answers to the second question (When you order lab tests do you think you often exceed, i.e., you order tests not addressed to that specific clinical presentation?) seem somewhat contradictory with the replies given to item n.1, since 120 (66\%)

\section{In case you answered "Yes" to the former question, what is the reason for this?}
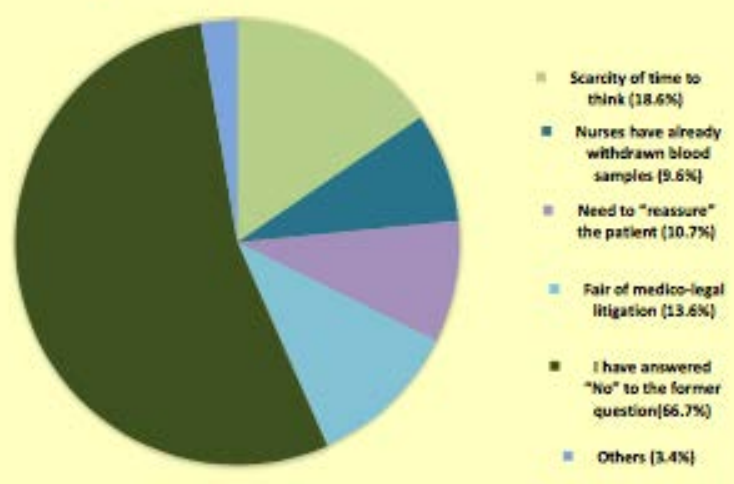

Figure 1. Responses to item n. 3.

\section{Do you think that exceeding in testing is:}

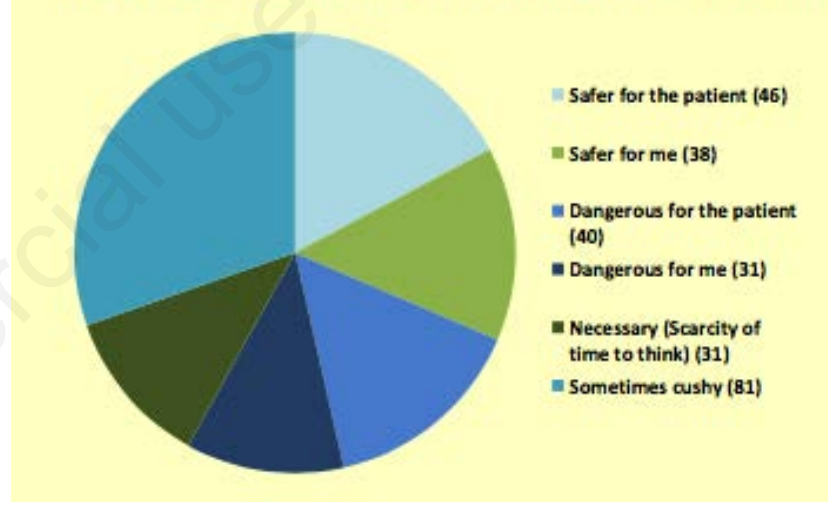

Figure 2. Responses to item n. 4 (absolute numbers).

\section{How do you keep yourself updated on new lab tests?}

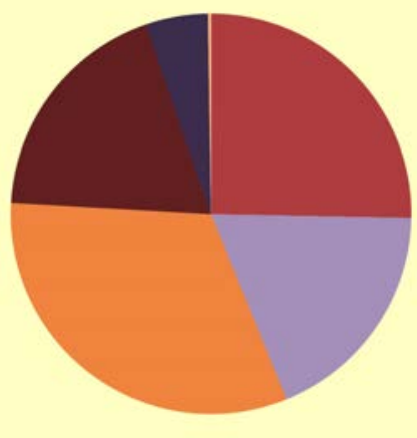

- Information sharing with colleagues (105)

$=$ Meetings and seminars (77)

= Guidelines and

recommendations (133)

a Scientific papers (78)

- WEB (even non medical sites) (21)

in Others (1)

Figure 3. Responses to item n. 7 (absolute numbers). 
responders selected Yes, whereas only 63 (34\%) selected No. It seems rather obvious that choosing panels intrinsically means exceeding with number of tests. Even more surprising was the reply to the following statement (Figure 1). A total number of 122 responders selected No to the former question, whereas only 63 actually provided a response, as previously noted. Notably, more EPs believe that this may be attributable to Shortage of time to think (18.6\%), than those who selected Fair of medical-legal litigation (13.6\%). Figure 2 shows the replies to question n. 4 Do you think that exceeding in testing is...In this case, the majority of responders selected Sometimes cushy, whereas other responses received similar attention, thus balancing the number of responders who think that exceeding with laboratory testing may be safer for patient and/or the doctor with those who believe that it could instead be dangerous. This specific question allowed multiple responses, so that the final sum is $>183$.

The replies to question n. 5 When you order a lab test do you usually consider if that test will give a response capable in influencing your clinical decision in that patient? were Always $(107 / 183 ; 58 \%)$ or Sometimes $(69 / 183 ; 38 \%)$, with only $7 / 183$ (4\%) EPs selecting Rarely $(5 / 183 ; 3 \%)$ or Never $(2 / 183 ; 1 \%)$. The question n. 6 was How much do you think to know the 'performance' of the tests you order? Overall, 107 (58.5\%) EPs answered Adequately, with an almost equally distributed response between Very well $(39 ; 21.3 \%)$ or Not adequately $(31 ; 16.9 \%)$, and only 6 $(3.3 \%)$ EPs selecting Insufficiently.

Figure 3 shows how the responders would be updated on new lab tests, displaying a large prevalence of Guidelines and recommendations (133/183).

A total number of $133(73.1 \%)$ responders selected Essential regarding the importance of the laboratory medicine in their clinical decisions, and 45 (24.7\%) selecting Moderate, whilst only 2 selected the options Scarce or Nothing at all (2 EPs did not respond).

The multiple answers to question 9 highlight an important evidence of potential appropriateness, since in a considerable number of Italian hospitals some obsolete tests ${ }^{15,16}$ are still included in the emergency panels (Figure 4).

When asked Which test do you consider the most important in influencing your clinical decisions?, nearly half of responders (89; $49 \%$ ) selected cardiac troponin, followed by hematologic testing (47; 26\%), and several others, as shown in Figure 5. Surprisingly, when asked Which test could you quit among those available in your hospital?, some responders affirmed that they could renounce to lipase $(3 ; 1.6 \%)$, and activated partial thromboplastin time $(2$; $1.1 \%)$. Then, when asked Which test do you wish to implement in emergency panels among those available in your hospital?, some responders replied that they would like to have lipase $(4 ; 2.2 \%)$, ample toxicology panel $(4 ; 2.2 \%)$, and white blood cell count ( 3 ; $1.6 \%$ ), thus raising the legitimate question as why these essential tests are still unavailable in some facilities.

Regarding the expenditure for laboratory testing, only 10 responders $(5.5 \%)$ replied that they knew exactly the costs of laboratory tests, whilst the vast majority of EPs affirmed that they were completely unaware $(51 ; 27.9 \%)$ or only partially aware $(122 ; 66.6 \%)$ of costs. The majority of responders to the following question $(138 ; 75.4 \%)$ replied that urgent laboratory tests may account between $20-50 \%$ of the total volume of analyses performed in the local laboratory service, approaching the real-world data, as previously published. ${ }^{17}$ Nevertheless, most responders substantially overestimated the real impact of laboratory expenditure on global hospital costs, whereby 120 responders $(65.5 \%)$ replied $10 \%(59 ; 32.2 \%)$ and $25 \%(61 ; 33.3 \%)$, being the real value about

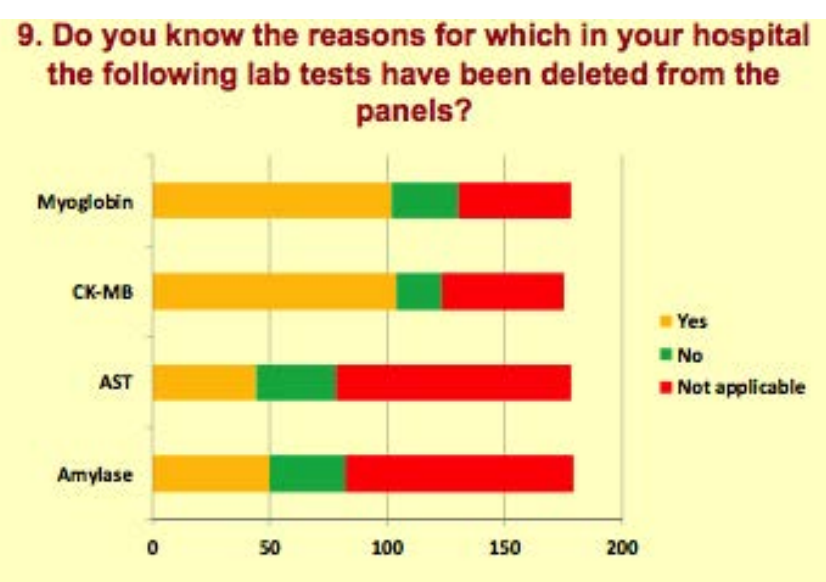

Figure 4. Responses to item n. 9 (absolute numbers). CK-MB, creatine kinase isoenzyme MB; AST, aspartate aminotransferase.

10. Which test do you consider the most important in influencing your clinical decisions?

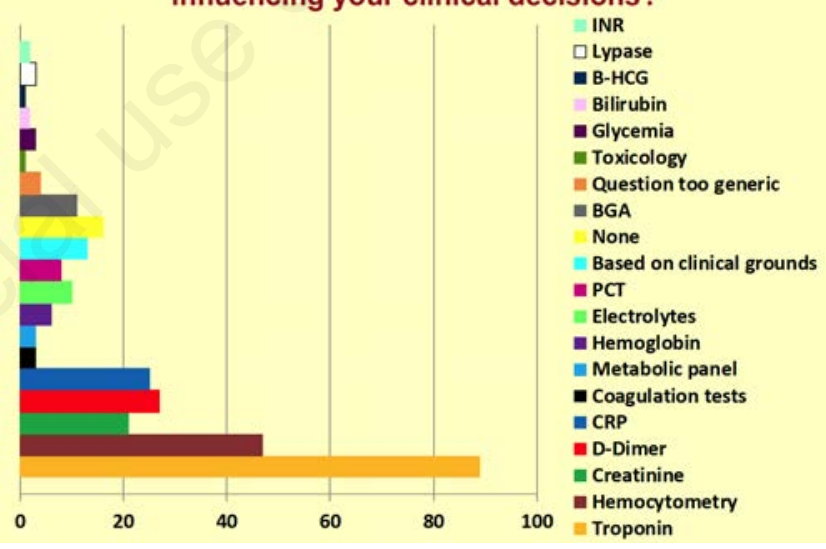

Figure 5. Responses to item n. 10. INR, international normalized ratio; B-HCG: beta-human chorionic gonadotropin; PCT, procalcitonin; CRP: C-reactive protein.

\section{I wish to go deeper in this field of knowledge:}

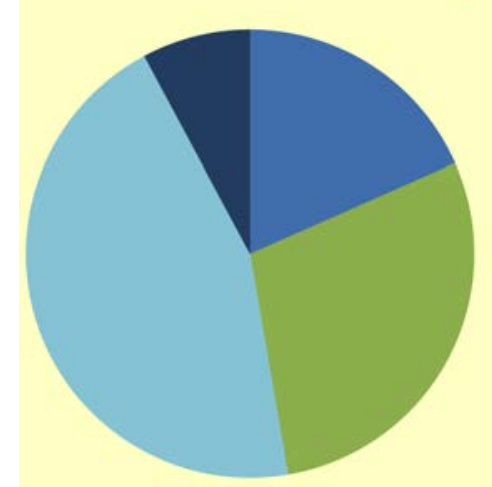

- To know the cost of single tests (54)

To better know the diagnostic performances (85)

To know the importance attributed by guidelines (132)

- To know the lab TAT of the tests (23)

Figure 6. Responses to item n. 17 (absolute numbers). TAT, turn around time. 
$2-3 \% .{ }^{18}$ When asked In which percentage do you think that lab tests influence the clinical management of each patient, in gener$a l$ ?, the vast majority of responders replied between $25-50 \%$ (55; $30 \%)$ and $50-75 \%(85 ; 46.5 \%)$. This is also in accordance with recent literature data, attesting that laboratory medicine would contribute to over $70 \%$ of clinical decisions. ${ }^{1}$

The responders to the survey also emphasized that they wish to go deeper in this field of knowledge by better knowing the importance attributed to the single laboratory tests by available guidelines (132), as well as the diagnostic performance of the tests (85) (percentage is not provided, since multiple choices were allowed). Other responses are summarized in Figure 6.

When asked In which diagnostic issues do you more frequently seek consultation with laboratory colleagues in test interpretation?, 88 responders $(48.1 \%)$, replied I never seek consultation with laboratory colleagues. Among those who occasionally seek consultation with laboratory colleagues, the most frequent field was hematology (35), followed by diagnostics of acute myocardial infarction (22) and coagulation disorders (20). When asked How could you describe your feedback from lab colleagues when you ask an expert opinion, the vast majority of responders replied that they find it useful (69: Almost always useful; 44: Sometimes use$f u l)$. Finally, when asked Would you consider useful to be engaged in meeting shared between EM and LM?, 147 (84.9\%) responders selected Yes.

\section{Discussion}

The results of this nationwide survey seem to confirm the existence of a suboptimal knowledge of both clinical and economic impact of urgent laboratory tests by Italian EPs. An unsatisfactory relationship between EPs and laboratory professionals seems also to emerge. On the other hand, it also interestingly emerged that some Italian hospitals still include obsolete o redundant tests in panels of urgent laboratory analyses (i.e., amylase, myoglobin, creatine kinase isoenzyme $\mathrm{MB}$; aspartate aminotransferase), which would actually contribute to increase the costs without producing tangible clinical benefit or even possibly misleading the diagnostic reasoning in the ED. $\cdot^{15,16}$

It has been convincingly demonstrated that the establishment of a virtuous circle between EM and LM would be essential for optimizing the use of human and technical resources in both environments, and is also necessary for improving clinical outcomes. ${ }^{19,20}$ Nevertheless, some major obstacles still challenge the establishment of this otherwise favorable liaison between EM and LM.

Defensive medicine is undoubtedly one of such issues, since the emergency settings are particularly plagued by malpractice and liability ${ }^{21}$ thus leading the way to an inappropriate utilization of laboratory resources. ${ }^{22}$ This unjustified practice carries several risks, such as the possible generation of false positive results, the disruption of laboratory organization and ultimate waste of human and economic healthcare resources. ${ }^{12}$

Previous evidence emphasized that the pattern of urgent test requesting in emergency settings is highly variable,,$^{13}$ thus underscoring the need to achieve widespread consensus for harmonizing requesting attitudes. The data obtained with our survey ultimately confirm these findings.

The ED is a challenging environment, probably one of the most complicated in the healthcare industry, where clinical decisions must be rapidly taken for diagnosing or ruling diseases, and hence assuring the best managed care, preventing litigations and overcrowding. In this complex scenario, correct utilization of laboratory resources seems essential. There is widespread perception, occasionally supported by personal belief of some laboratory professionals, that diagnostic testing may be a foolproof instrument which can help diagnosing everything. Although both volume and quality of laboratory tests have enormously increased during the past decade,,$^{23-25}$ no single test is capable to accurately define a healthy status, so that diagnostic testing only prescribed to satisfy patient's wishes should be eluded.

Although many physicians, including those working in the ED, have adequate knowledge of diagnostic implications of laboratory analyses, they frequently ignore the inherent drawbacks and limitations of such tests, especially for recently introduced biomarkers. These typically encompass technical and clinical aspects, which may ultimately derange the clinical efficiency of diagnostic testing. Although relatively infrequent, the results of some tests may then be occasionally unexpected or frankly contradictory with the patient status. Whenever biological causes can be ruled out, the more reasonable justification is a preanalytical error, such as misidentification, analysis of unsuitable specimens (for quality or quantity), as well as the presence of possible analytical interference due to the presence of rheumatoid factor, heterophilic antibodies, undue clots, etc. ${ }^{26,27}$ Although it is clearly unlikely that EPs can stay abreast of each single problem occurring during sample collection and handling, whenever test results conflict with clinical signs and symptoms, the effect of preanalytical variability shall always be investigated. In this setting, favorable collaboration between EM and LM will be effective to solve most of the problems and eventually detect the most likely cause for the bias.

\section{Conclusions}

In conclusion, the virtuous circle between EM and LM, promoted and partially implemented by the two national societies AcEMC and SIBioC, should be further reinforced for optimizing clinical pathways in Italian EDs, for improving clinical outcomes and reducing unnecessary expenditures. Major agreement among laboratory professionals for harmonization of urgent test panels seems advisable. ${ }^{28}$

\section{References}

1. Hallworth MJ. That '70\%' claim again. Ann Clin Biochem 2018;55:517-8.

2. Lippi G. The irreplaceable value of laboratory diagnostics: four recent tests that have revolutionized clinical practice. EJIFCC 2019;30:7-13.

3. Lippi G, Guidi GC. The power of negative thinking. Am J Emerg Med 2008;26:373-4.

4. Lippi G, Panteghini M, Bernardini S, et al. Laboratory testing in the emergency department: an Italian Society of Clinical Biochemistry and Clinical Molecular Biology (SIBioC) and Academy of Emergency Medicine and Care (AcEMC) consensus report. Emerg Care J 2017;13:6600.

5. Lippi G, Balboni F, Bonfanti L, et al. Laboratory Medicine and Emergency Medicine: an essential partnership. Biochim Clin 2018;42:335-42.

6. Casagranda I, Cavazza M, Clerico A, et al. Proposal for the use in emergency departments of cardiac troponins measured with 
the latest generation methods in patients with suspected acute coronary syndrome without persistent ST-segment elevation. Clin Chem Lab Med 2013;51:1727-37.

7. Lippi G, Montagnana M, Balboni F, et al. Academy of Emergency Medicine and Care-Society of Clinical Biochemistry and Clinical Molecular Biology consensus recommendations for clinical use of sepsis biomarkers in the emergency department. Emerg Care J 2017;13:6877.

8. Lippi G, Cervellin G, Casagranda I, et al. D-dimer testing for suspected venous thromboembolism in the emergency department. Consensus document of AcEMC, CISMEL, SIBioC, and SIMeL. Clin Chem Lab Med 2014;52:621-8.

9. Lippi G, Cervellin G, Plebani M. The ten commandments of laboratory testing for emergency physicians. Clin Chem Lab Med 2014;52:183-7.

10. Lippi G, Plebani M. False myths and legends in laboratory diagnostics. Clin Chem Lab Med 2013;51:2087-97.

11. Hardin E. Emergency medicine and the laboratory. J Natl Med Assoc 1996;88:279-82.

12. Lippi G, Bovo C, Ciaccio M. Inappropriateness in laboratory medicine: an elephant in the room? Ann Transl Med 2017;5:82.

13. Salinas M, Lòpez-Garrigòs M, Uris J. Differences in laboratory requesting patterns in emergency departments in Spain. Ann Clin Biochem 2013;50:353-9.

14. Carraro P. Recommendations for appropriate laboratory tests in emergency department typical conditions. Biochim Clin 2011;35:207-28.

15. Wu AHB, Lewandroski K, Gronowski AM, et al. Antiquated tests within the clinical pathology laboratory. Am J Manag Care 2010;16:220-7.

16. Alvin MD, Jaffe AS, Ziegelstein RC, et al. Eliminating Creatine Kinase-Myocardial Band testing in suspected acute coronary syndrome. A value-based quality improvement. JAMA Intern Med 2017;177:1508-12.
17. Lippi G, Mattiuzzi C, Plebani M. Stat testing utilization in clinical laboratories. National survey of Italian Society of Clinical Biochemistry and Molecular Biology (SIBioC). Clin Chem Lab Med 2014;52:e79-84.

18. Lippi G. Weighting healthcare efficiency against available resources: value is the goal. Diagnosis (Berl) 2018;5:39-40.

19. Plebani M, Lippi G. Closing the brain-to-brain loop in laboratory testing. Clin Chem Lab Med 2011;49:1131-3.

20. Lippi G, Cervellin G. From laboratory instrumentation to physician's brain calibration: the next frontier for improving diagnostic accuracy? J Lab Prec Med 2017;2:74.

21. Cervellin G, Cavazza M. Defensive medicine in the emergency department. The clinicians' perspective. Emerg Care J 2016;12:561.

22. Montagnana M, Lippi G. The risks of defensive (emergency) medicine. The laboratory perspective. Emerg Care J 2016;15:581.

23. Lippi G, Plebani M. Biomarker research and leading causes of death worldwide: a rather feeble relationship. Clin Chem Lab Med 2013;51:1691-3.

24. Favaloro EJ, Lippi G. Recent advances in mainstream hemostasis diagnostics and coagulation testing. Semin Thromb Hemost 2019;45:228-46.

25. Plebani M, Laposata M, Lippi G. Driving the route of laboratory medicine: a manifesto for the future. Intern Emerg Med 2019;14:337-40.

26. Lippi G, Becan-McBride K, Behúlová D, et al. Preanalytical quality improvement: in quality we trust. Clin Chem Lab Med 2013;51:229-41.

27. Lippi G, Plebani M, Di Somma S, et al. Hemolyzed specimens: a major challenge for emergency departments and clinical laboratories. Crit Rev Clin Lab Sci 2011;48:143-53.

28. Lippi G, Mattiuzzi C. Project management in laboratory medicine. J Med Biochem 2019;38:401-6. 